\title{
Diffusion and magnetic field effects on stellar surfaces
}

\author{
Oleg Kochukhov
}

Institut für Astronomie, Universität Wien, Türkenschanzstraße 17, 1180 Wien, Austria

email: kochukhov@astro.univie.ac.at

\begin{abstract}
Chemically peculiar stars are ideal astrophysical laboratories for furnishing a wide range of theoretical and observational aspects of our knowledge of the physical processes in stars. Recent dramatic improvements in the quality of the observational data and refinements of the modelling techniques led to an emergence of a new branch of stellar astrophysics which is focused on the reconstruction and the understanding of the origin of the three-dimensional structures in stellar surface layers. In this contribution I present an overview of recent results of the detailed modelling of the chemical nonuniformities, the magnetic and the pulsation velocity fields in the atmospheres of A stars. New Doppler imaging analyses of the magnetic field and the chemical inhomogeneities reveal an unexpected complexity of the surface formations and suggest that nonmagnetic phenomena play an important role in shaping the geometry of chemical spots. Consideration of the line profile shapes observed at high spectral and time resolution has made it possible to probe the radial dependence of the chemical abundances and the pulsation characteristics of cool pulsating Ap stars. An extension of Doppler mapping to the reconstruction of non-radial stellar oscillation structure delivers a solution of the long-standing problem of the pulsational geometry of roAp stars and helps to elucidate the interrelation between the pulsations, the magnetic field and the stellar rotation.
\end{abstract}

Keywords. Stars: magnetic fields, stars: chemically peculiar, stars: oscillations, stars: spots, stars: individual ( $\alpha$ And, $\alpha^{2}$ CVn, $\alpha$ Cir, 53 Cam, HR 3831)

\section{Introduction}

The investigation of stellar magnetism and its influence on the stellar surfaces and the envelopes is a quickly maturing research area. In the past different observational aspects of A stars, such as the stellar rotation, the atmospheric structure, the magnetic field and the chemical inhomogeneities induced by radiative diffusion, were considered separately, using simple phenomenological models. In contrast, quite a few recent observations and modelling efforts established a very close and sometimes surprising connection between the different phenomena observed in the peculiar A stars. These important developments emphasize the necessity for self-consistent models based on elaborate theoretical foundations and suggest that we are witnessing the emergence of a new branch of stellar astrophysics focused on the comprehensive analysis of the origin and the evolution of 3-D formations in stellar atmospheres.

In this contribution I highlight the results of some recent analyses of the complex interrelations between the magnetic field, the chemical structures and the non-radial pulsation in the atmospheres of CP stars. I focus on the studies which concentrated on the detailed modelling of the shapes and the variability of stellar line profiles. 


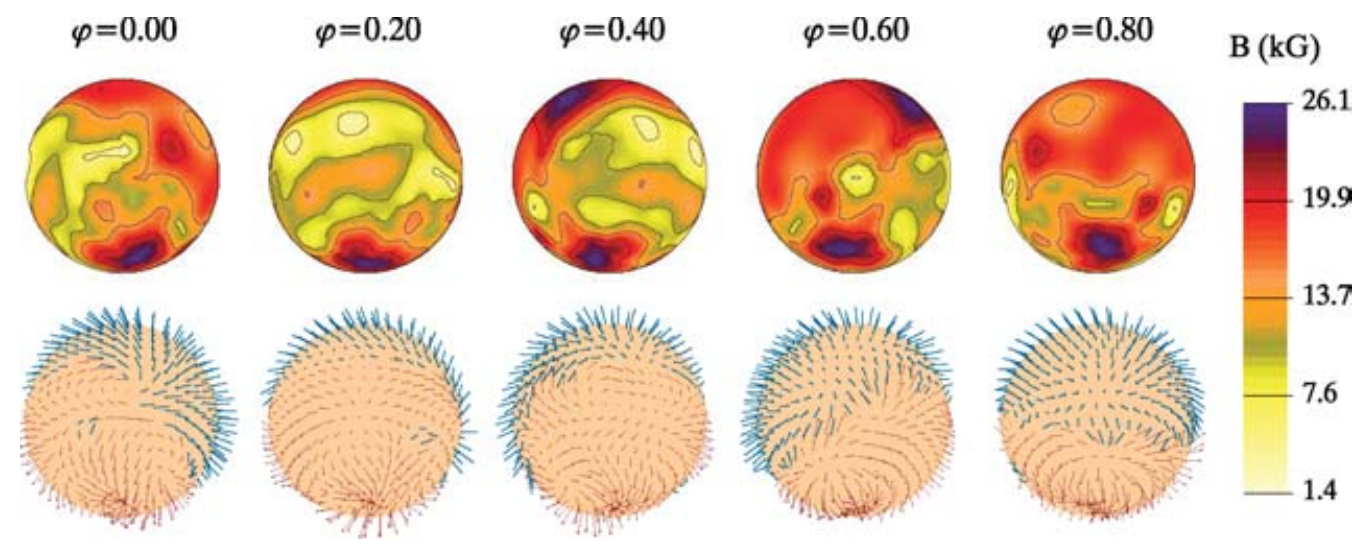

Figure 1. Distribution of the magnetic field strength (upper panel) and the field orientation (lower panel) over the surface of $53 \mathrm{Cam}$ as derived from the Stokes IQUV profiles of three Fe II lines. The star is shown at five equidistant rotation phases as indicated at the top of the figure. The aspect corresponds to the inclination angle $i=123^{\circ}$ and a vertically oriented rotation axis.

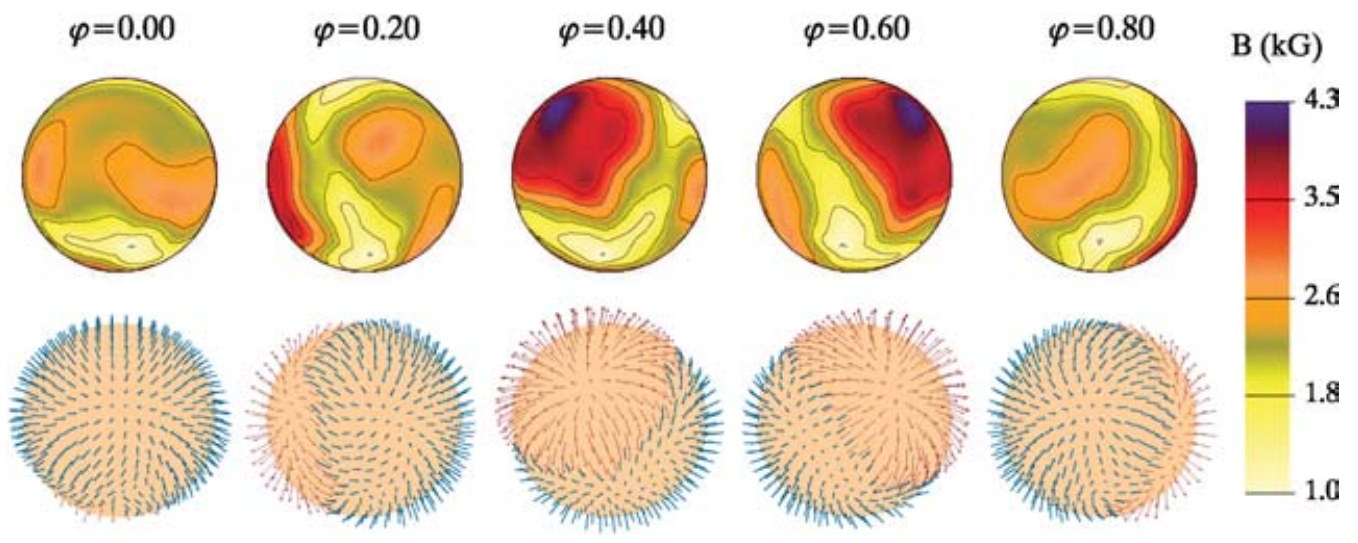

Figure 2. Same as Fig. 1 for the maps of the magnetic field strength (upper panel) and field orientation (lower panel) derived for $\alpha^{2} \mathrm{CVn}$ using the Stokes IQUV profiles of two lines of Fe II and one line of $\mathrm{Cr}$ II. The inclination angle of the stellar rotation axis is $i=130^{\circ}$.

\section{Magnetic field topologies}

For many decades studies of the line polarization in the magnetic stars were limited to the Stokes $I$ and $V$ spectra and relied on a statistical multi-line analysis carried out under a number of restrictive approximations (e.g. Mathys 1991). This situation changed drastically a few years ago with the possibility of acquiring high-resolution spectra in all four Stokes parameters. The most important contribution came from the MuSiCoS spectropolarimeter which was used to obtain the first systematic 4 Stokes parameter observations of the magnetic CP stars (Wade et al. 2000). To match this improvement in the quality of the data, several groups have developed computer codes for realistic synthesis of the stellar polarization spectra (Wade et al. 2001). A more challenging problem of the magnetic inversion, a reconstruction of the stellar magnetic geometry from the Stokes parameter observations, was addressed by Piskunov \& Kochukhov (2002). The magnetic Doppler imaging code that we have developed makes it possible to derive magnetic maps self-consistently with the distributions of the chemical elements. Most importantly, in 
mapping the magnetic geometry we do not use a multipolar field model and, thus, attempt to reconstruct the vector field distribution making no a priori assumptions.

The first inversion of the stellar high-resolution spectra in all 4 Stokes parameters was carried out for the well-known magnetic CP star 53 Cam (Kochukhov et al. 2004a). Bagnulo et al. (2001) demonstrated that the previous multipolar models of the magnetic field in this star were unable to reproduce the polarization profiles observed with $\mathrm{Mu}-$ SiCoS. Even a direct fit of a general dipole plus quadrupole superposition to the Stokes parameter observations fails to match the low amplitude of the linear polarization signal. The failure of such a simple magnetic distribution suggests that the magnetic topology is considerably more complicated. Kochukhov et al. (2004a) obtained a satisfactory model of the field in 53 Cam using magnetic Doppler imaging. With this method the magnetic map has been reconstructed directly from the Stokes $I Q U V$ parameters, without relying on a low-order multipolar description of the field. This model successfully reproduces the Stokes parameter spectra and the other magnetic observables of $53 \mathrm{Cam}$.

The resulting map of the field strength (Fig. 1) appears to be strikingly complex. However, the overall distribution of the field orientation is still dominated by just two large regions of inward and outward directed field. What are the physical effects which gave rise to such a field distribution and how typical is it for other magnetic stars? At the moment only one additional star, $\alpha^{2} \mathrm{CVn}$, has been analysed in all 4 Stokes parameters (Kochukhov et al. , in preparation). When we applied the same inversion technique as was employed for $53 \mathrm{Cam}$, a somewhat less structured magnetic map was found sufficient to fit the Stokes spectra of $\alpha^{2} \mathrm{CVn}$. The respective spherical maps of the magnetic field strength and orientation are illustrated in Fig. 2. A comparative numerical analysis of the magnetic maps derived for the two stars confirms the impression that the magnetic field in $\alpha^{2} \mathrm{CVn}$ is simpler. Thus, we find that some CP stars possess a much more complicated field geometry than commonly believed. At the same time, there also seems to exist a large intrinsic scatter in the level of field complexity among different magnetic stars.

\section{Surface chemical structures}

In contrast to the Stokes parameter inversion, abundance Doppler mapping is a wellknown tool, which has been applied to spotted Ap stars for many years. Recent progress in this field stems from improvements in the numerical methods and in the quality of observational material. These two factors allow one to construct more accurate chemical maps for a larger number of elements. Recently Kochukhov et al. (2004b) completed a very detailed abundance Doppler imaging study of the roAp star HR 3831. Our analysis is the most thorough examination of the surface chemical patterns for an Ap star. Maps of 17 chemical elements were recovered. Some of these distributions are based on the exceedingly high quality spectra collected by Kochukhov \& Ryabchikova (2001b) ( $R=$ $123000, S / N>500,36$ rotation phases), which enabled us to explore the surface spot pattern down to the fundamental resolution limit of Doppler imaging.

Fig. 3 shows an example of the chemical maps reconstructed for HR 3831. Only for a few elements do we find an obvious correlation with the magnetic geometry. For instance, $\mathrm{Li}$ seems to be accumulated at the poles of a dipolar field and $\mathrm{O}$ is enhanced at the magnetic equator. These nicely symmetric distributions are exceptional. Almost all other elements, for instance iron (Fig. 3), are distributed in a rather complex manner and do not follow the symmetry of the dominant dipolar magnetic field component of HR 3831.

$\mathrm{Ba}$ has one of the most high-contrast distributions. This element is concentrated in a series of very small spots which are found at the magnetic poles and at the intersection of the magnetic and the rotation equators. The geometry of the surface chemical patterns is 

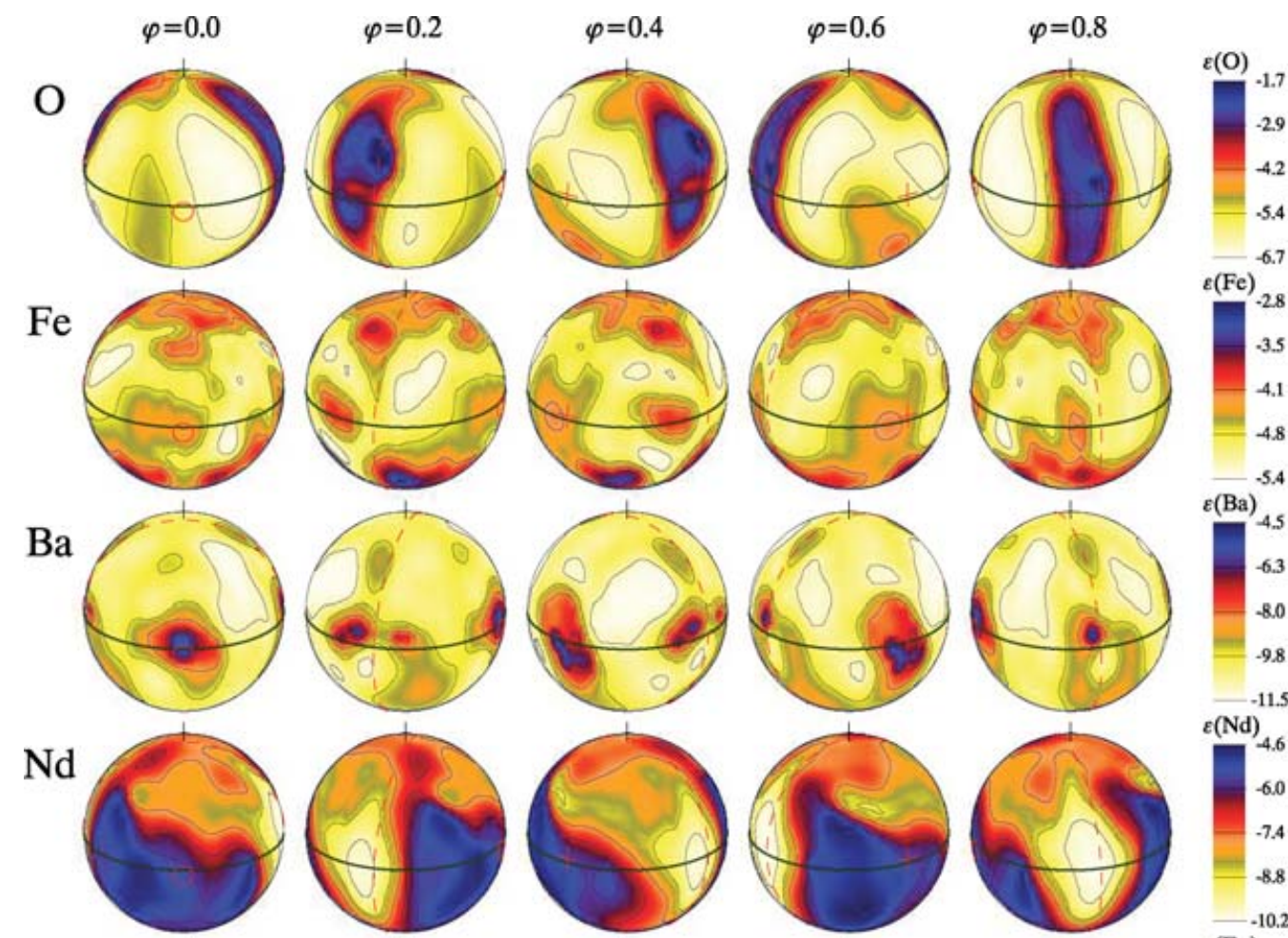

$\varepsilon(\mathrm{Ba})-4.5$

$\mathrm{Eu}$
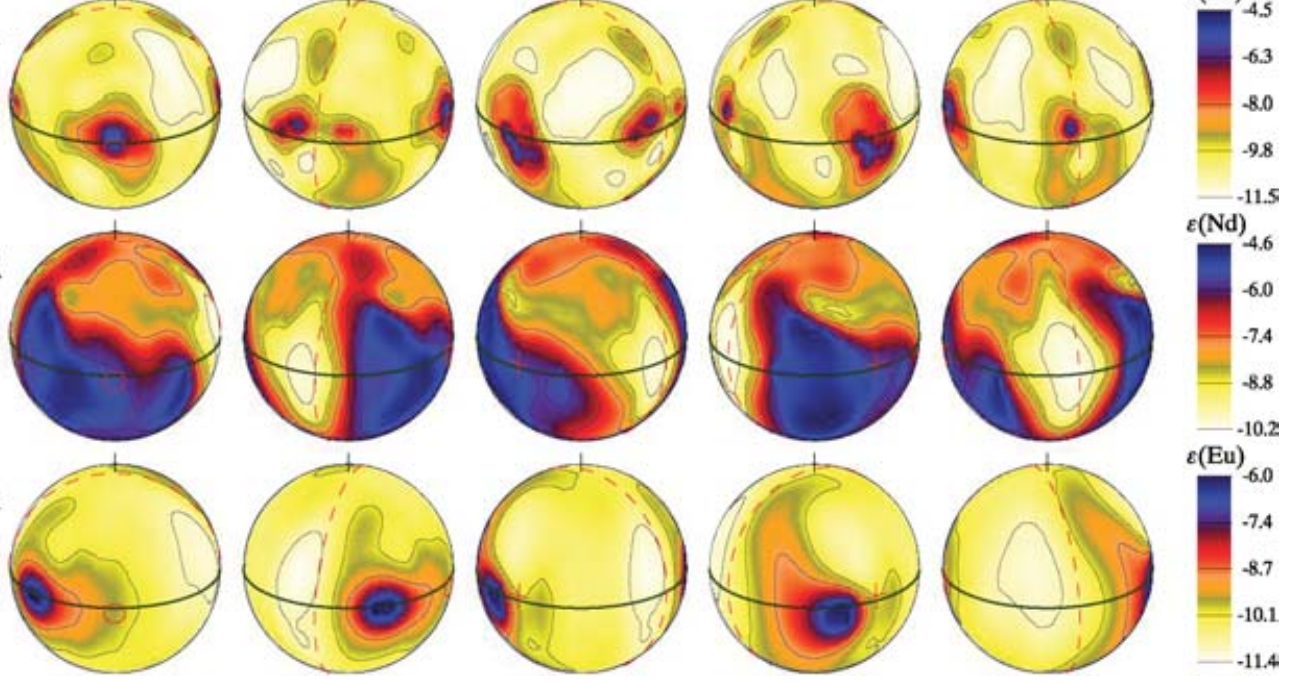

Figure 3. Surface distributions of $\mathrm{O}, \mathrm{Fe}, \mathrm{Ba}, \mathrm{Nd}$ and $\mathrm{Eu}$ reconstructed for the roAp star HR 3831. The star is shown at five equidistant rotation phases at the inclination angle $i=68^{\circ}$ and a vertically oriented axis of rotation. In the spherical plots darker areas correspond to higher element concentration. The thick solid line shows the location of the rotational equator, whereas the dashed line, plus sign and circle correspond, respectively, to the magnetic equator and poles of the dipolar magnetic field in HR 3831.

extremely diverse. Even elements with similar atomic weights often show vastly dissimilar maps. It was commonly believed that all rare-earth elements have approximately similar surface distributions and are concentrated at the magnetic poles. This is certainly not true for HR 3831. Eu shows small spots, but they are noticeably displaced from the magnetic poles. On the other hand, $\mathrm{Nd}$ avoids the magnetic equator, but is present elsewhere at the surface. These results demonstrate the complexity of the diffusion in Ap stars and discard a naïve view of the chemical spots and rings symmetric with respect to magnetic field. Abundance distributions are clearly affected by a range of poorly understood phenomena, which are not directly related to magnetic field.

More compelling evidence for the nonmagnetic modulation of the radiative diffusion has emerged with the discovery of surface inhomogeneities in the brightest $\mathrm{Hg}-\mathrm{Mn}$ binary star $\alpha$ And. Hg-Mn stars are known to be nonmagnetic (Shorlin et al. 2002), and $\alpha$ And is not an exception from this rule. Recent spectropolarimetric observations by Wade et al. (2004) find no longitudinal field above $50 \mathrm{G}$ and establish an upper limit of the global field 
at about $300 \mathrm{G}$, which is roughly an equipartition field for $\alpha$ And. Yet, Adelman et al. (2002) reported convincing observations of the variability of the resonance $\mathrm{Hg}$ II $\lambda 3984$ line and reconstructed the surface distribution of mercury. The concentration of this element was found to increase from the rotation poles towards the equator. A clear trend of abundance versus latitude may be connected with the change of surface gravity and resulting latitudinal modulation of the diffusion processes due to a fairly rapid rotation of $\alpha$ And. This does not explain the origin of the longitudinal abundance variation, which is observed as a series of 4 spots of $\mathrm{Hg}$ overabundance at or near the equator. Perhaps, the longitudinal structure is caused by the dynamic tides induced by the secondary star in the $\alpha$ And system, or it may be a signature of an undetected weak magnetic field. In any case, the surprising $\alpha$ And phenomenon shows how incomplete is our understanding of diffusion even when we deal with the brightest nonmagnetic stars.

\section{Spectroscopic signatures of non-radial pulsation}

The most outstanding characteristic of the spectroscopic pulsational variability in magnetic Ap stars is the diversity of pulsational changes observed in lines of different elements. Pulsations are clearly detected in lines of singly and doubly ionized rare-earth elements (REE) but typically do not show up in other lines (e.g., Kochukhov \& Ryabchikova 2001a). Time-resolved radial velocity (RV) analyses demonstrated differences of almost a factor of 100 in amplitude and up to $180^{\circ}$ in phase between the variability of different lines. Nothing like that is observed in normal Main Sequence pulsating stars. It was anticipated that the surface abundance spots may be responsible for this diversity of pulsational behaviour. However, Doppler mapping of the roAp star HR 3831 (Kochukhov et al. 2004b) proved this hypothesis to be wrong. The chemical elements showing maximal pulsational variability are not distributed over the surface in any special way.

Looking for an explanation of the puzzling roAp pulsation signature, we have established a clear relation between the oscillations and the stratification of the chemical abundances (Ryabchikova et al. 2002). All lines with high pulsation amplitude belong to REEs, which are affected by an extreme stratification and are probably formed high in the atmospheres of roAp stars. On the other hand, light and iron-peak elements tend to be concentrated in deep layers and show little pulsational variability. Thus, chemical stratification acts as a spatial filter for observations of the pulsational fluctuations and allow us to resolve the vertical structure of $p$-modes. This is done with the pulsation tomography technique, which is a method to study depth dependences of the pulsation properties using lines formed at different depth. A key point here is that the line formation depths in roAp stars are strongly modified by the chemical gradients. Therefore, the derivation of the vertical chemical profiles is a crucial ingredient of the roAp pulsational analysis.

Fig. 4 demonstrates an example of the application of the pulsation tomography method to the roAp star $\gamma$ Equ. The left panel shows the pulsation RV amplitude at the formation depth of the selected spectral lines and the RV bisector measurements at different levels of the $\mathrm{H} \alpha$ core. A recent analysis by Mashonkina et al. (2005) indicated that NLTE effects become significant for the REEs concentrated in the upper atmospheric layers. They found that the bottom of the REE cloud and the formation depth of the corresponding lines is shifted to about $\log \tau_{5000}=-4$ when NLTE effects are accounted for. This leaves us with a very clear picture of a rapid increase of the pulsation amplitude towards the upper atmosphere of roAp stars. This behaviour has no theoretical explanation so far.

Interpretation of the pulsation phases is a more difficult task since it crucially depends on the accuracy that we can achieve in modelling the difference between individual REE 

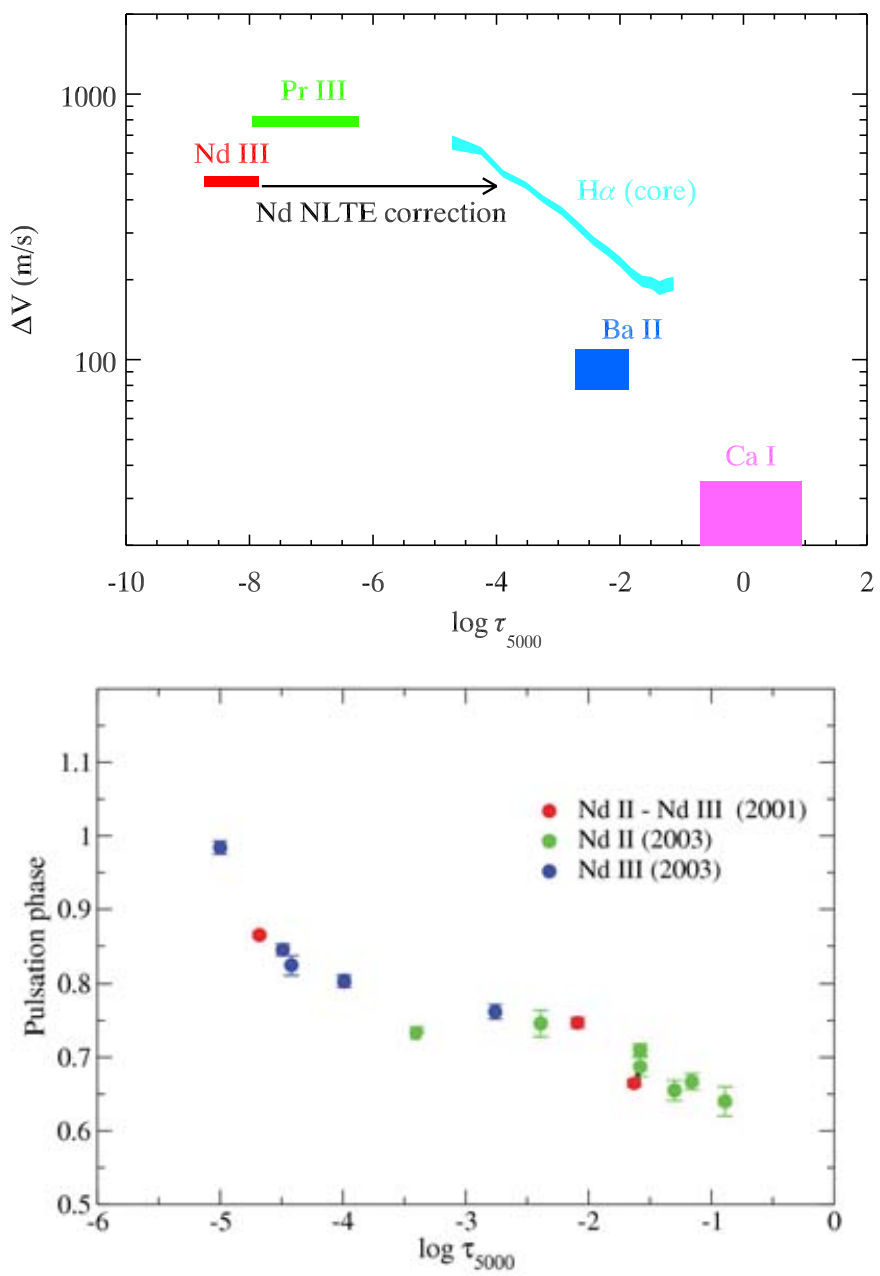

Figure 4. Pulsation tomography reconstruction of the vertical pulsation mode structure in the roAp star $\gamma$ Equ. Left panel: pulsation amplitude as a function of optical depth derived from the $\mathrm{RV}$ variation of metal lines and bisector measurements in the $\mathrm{H} \alpha$ core. Right panel: pulsation phase as a function of the formation depth of $\mathrm{Nd}$ II and $\mathrm{Nd}$ III lines calculated taking into account NLTE effects and the vertical stratification of $\mathrm{Nd}$.

lines. It appears that a NLTE analysis is compulsory for revealing the variation of pulsation phase with depth. The right panel in Fig. 4 shows results of the calculation for neodymium in $\gamma$ Equ (Mashonkina et al. 2005). The relative phase of the RV variation is plotted as a function of formation depth calculated taking into account stratification and NLTE effects. There is a convincing evidence for the depth-dependence of pulsation phase. This means that in the REE-rich atmospheric layers of $\gamma$ Equ we observe a running pulsation wave propagating upwards.

\section{Surface mapping of stellar non-radial pulsations}

The vertical diffusion has a very prominent effect on the oscillations and enables us to recover the vertical mode structure. This information has to be complemented by the horizontal picture to construct a comprehensive 3-D map of the pulsational disturbances and to understand the interaction between the global magnetic field and the non-radial 

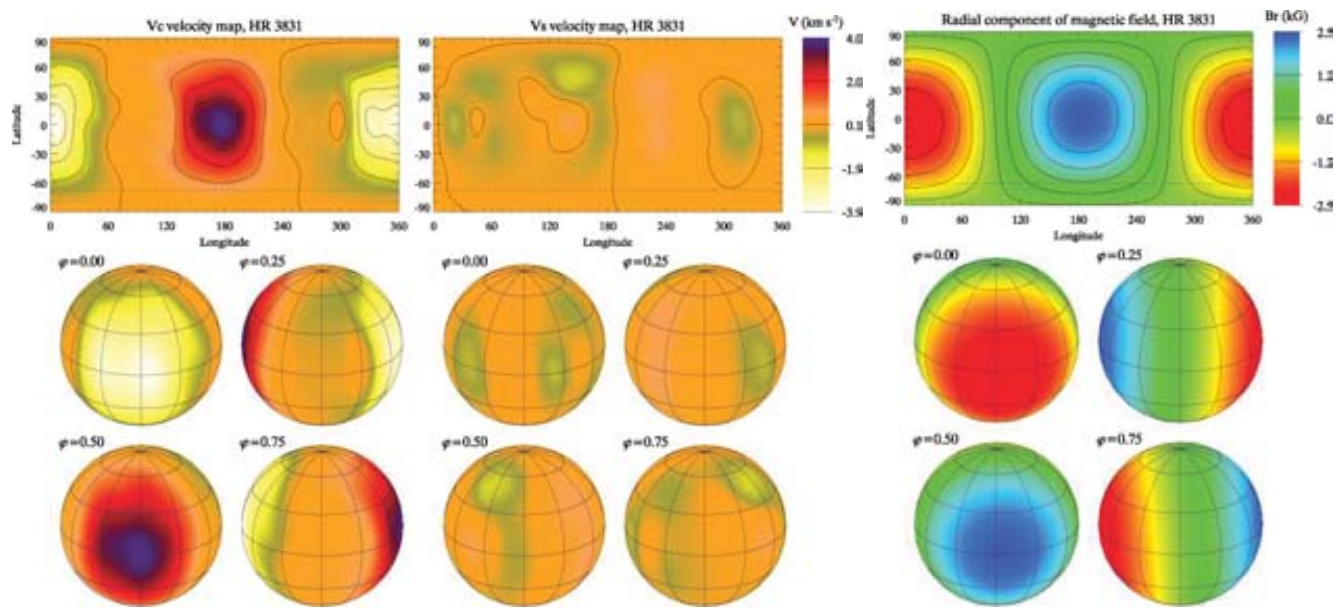

Figure 5. The first Doppler image of a stellar non-radial pulsation velocity field. Left and center: rectangular and spherical projections of the pulsation velocity amplitude maps derived for HR 3831. Right: distribution of the vertical component of magnetic field in HR 3831.

pulsations. This is a classical problem for roAp research. However, the traditional photometric time-series observations are not informative enough to tell us any details beyond a rather unspecific inference that the pulsations are probably oblique and are roughly described by axisymmetric dipolar modes (e.g., Kurtz 1990). Spectroscopic modelling is the key to resolving the horizontal mode structure and verifying conflicting theoretical predictions of the distortion of pulsation modes by the magnetic field and the rotation.

A recent breakthrough in understanding the roAp pulsation geometry has been achieved with the help of Doppler mapping of the pulsation velocity field. I developed a new Doppler imaging code (Kochukhov 2004, 2005) to recover the non-radial pulsation structure directly from the variability of line profiles. The new inversion procedure takes into account surface chemical inhomogeneities and does not assume a spherical harmonic basis for pulsation. To describe a time-dependent pulsation field, I represent each component of the velocity vector with the two constant surface maps, e.g., $V_{r}(t, \theta, \varphi)=V_{r}^{C}(\theta, \varphi) \cos (\omega t)+V_{r}^{S}(\theta, \varphi) \sin (\omega t)$. This is equivalent to mapping distribution of both pulsation amplitude and phase over the stellar surface.

The new pulsation mapping technique was applied to the high-resolution time series observations of the Nd III $\lambda 6145$ line in the spectrum of HR 3831. It was possible to obtain a satisfactory fit to the profile variability with only the vertical pulsation displacement. Fig. 5 shows the rectangular and the spherical projections of the first Doppler image of the stellar pulsation velocity and compares the pulsation and the magnetic field maps. These surface images show that the pulsations are clearly inclined with respect to the rotation axis. This is the first direct evidence for oblique pulsations in stars. Furthermore, the obliquity angle is close to $90^{\circ}$, the pulsations are almost axisymmetric and the pulsation axis coincides with the axis of the dipolar magnetic component. This is, essentially, the standard oblique pulsator model of Kurtz (1982) suggested long time ago. However, pulsation mapping derives this structure directly from the observations, whereas previously it was adopted ad hoc.

A closer look at the pulsation maps reveals some nontrivial and significant deviations from a pure oblique dipolar mode geometry. Pulsations are found to be strongly confined to the magnetic poles. Harmonic expansion in the magnetic reference frame demonstrates that the pulsation structure of HR 3831 can be roughly reproduced with a superposition 
of $\ell=1$ and $\ell=3$ axisymmetric components, in a very good agreement with the recent theoretical prediction by Saio \& Gautschy (2004). On the other hand, the nonaxisymmetric $\ell=1$ components are not needed for the description of pulsations in HR 3831 . The revised oblique pulsator model of Bigot \& Dziembowski (2002) predicted such components as a result of the influence of stellar rotation. These authors also argued that pulsations are not aligned with magnetic field. Neither prediction is confirmed, hence the model of Bigot \& Dziembowski (2002) is not applicable to HR 3831. This can be taken as an indication that the magnetic field has a much stronger effect on the mode geometry compared with the stellar rotation.

\section{Acknowledgements}

I would like to acknowledge the Lise Meitner fellowship granted by the Austrian Science Fund (project No. M757-N02).

\section{References}

Adelman, S. J., Gulliver, A. F., Kochukhov, O. P., \& Ryabchikova, T. A. 2002, ApJ, 575, 449

Bagnulo, S., Wade, G. A., Donati, J.-F., et al. 2001, A\&BA, 369, 889

Bigot, L., \& Dziembowski, W. A. 2002, A\& A, 391, 235

Kochukhov, O., \& Ryabchikova, T. 2001a, A\&\&A, 374, 615

Kochukhov, O., \& Ryabchikova, T. 2001b, A\&A, 377, L22

Kochukhov, O., Piskunov, N., Ilyin, I., Ilyina, S., \& Tuominen, I. 2002, A\& A, 389, 420

Kochukhov, O. 2004, A\& A in press

Kochukhov, O., Bagnulo, S., Wade, G. A., et al. 2004a, A\&A, 414, 613

Kochukhov, O., Drake, N. A., Piskunov, N., \& de la Reza, R. 2004b, A\&\&A in press

Kochukhov, O. 2005, These Proceedings, GP20

Kurtz, D. W. 1982, MNRAS, 200, 807

Kurtz, D. W. 1990, ARAEA, 28, 607

Mashonkina, L., Ryabchikova, T., \& Ryabtsev, A. 2005, These Proceedings, 315

Mathys, G. 1991, $A \mathscr{E} A S, 89,121$

Piskunov, N., \& Kochukhov, O. 2002, A\& $\mathcal{G}$ A, 381, 736

Ryabchikova, T., Piskunov, N., Kochukhov, O., et al. 2002, A\&\&A, 384, 545

Saio, H., \& Gautschy, A. 2004, MNRAS, 350, 485

Shorlin, S. L. S., Wade, G. A., Donati, J.-F., et al. 2002, A\&A A, 392, 637

Wade, G. A., Donati, J.-F., Landstreet, J. D., \& Shorlin, S. L. S. 2000, MNRAS, 313, 823

Wade, G. A., Bagnulo, S., Kochukhov, O., et al. 2001, A\&\&A, 374, 265

Wade, G. A., Abecassis, M., Auriere, M., et al. 2004, in Magnetic Stars 2003, eds. Yu. V. Glagolevskij and I.I. Romanyuk, in press

\section{Discussion}

RYABCHIKOVA: Do you think that the more complex topology of the magnetic field in HR 3831, compared to that of 53 Cam, may explain the diversity of abundance maps?

Kochukhov: There is no indication that the field in HR 3831 is substantially more complicated than a dipole. On the other hand, we clearly see that a complex field can coexist with a fairly simple abundance structures in $53 \mathrm{Cam}$, whereas small-scale spots in HR 3831 seem to have no counterpart in the magnetic geometry. Thus, a relation between magnetic and chemical structures is not as straightforward as it was commonly believed.

Mathys: How do you deal with the isotopic effect in mapping $\mathrm{Hg}$ on the surface of $\alpha$ And? 
Kochukhov: In mapping the $\mathrm{Hg}$ distribution on $\alpha$ And we recovered the surface distribution of the isotope mixture using the classical $q$-parameterization. We found no evidence for large surface variations of the isotope composition.

ALECIAN: Is there any indication of changes of the magnetic topology with time? In other words, have you observed the same star after one or two years to see if the reconstructed structures are the same?

Kochukhov: The first magnetic maps of Ap stars derived from all 4 Stokes parameters were based on a unique observational material collected over the time-span of 3-4 years. We had no chance to collect two or more independent datasets for any of the targets. Nevertheless, we are certainly planning to reobserve 53 Cam to check the stability of the small-scale magnetic structures detected with our Stokes inversion technique.

Michaud: When we made a model for 53 Cam, Babel and I could reproduce the time averaged abundances observed in that star with no arbitrary parameters, using only the observed magnetic field and the known stellar properties. However, Babel had to introduce a mass loss at the magnetic poles to reproduce approximately the then observed spots on 53 Cam. I do not know how this should be changed by the much more precise magnetic and abundance maps now available.

Kochukhov: The abundance distributions of 53 Cam derived with new techniques do not differ considerably from the earlier maps. The major change is in the field structure. However, despite the complexity of the field, it is still dominated by the two large regions of inward and outward directed magnetic flux, which is probably the most relevant for confining the mass loss. Thus, it may not be necessary to modify the theoretical diffusion model dramatically. But it is certainly worth continuing the work you have initiated with Babel and consider these diffusion calculations in the context of the new surface maps. 